\section{Orofacial granulomatosis in children can be the initial manifestation of systemic disease: a presentation of two cases}

\author{
Anne Birgitte Simonsen, Mette Deleuran \\ Department of Dermatology, Aarhus \\ University Hospital, Aarhus, Denmark
}

\begin{abstract}
Orofacial granulomatosis is a chronic granulomatous condition characterized by relapsing and remitting lip swelling and oral involvement that may include deep ulcers, tags and cobblestone formation. It occurs as an independent entity but also in conjunction with systemic diseases such as tuberculosis, sarcoidosis and Crohn's disease. The clinical presentation is not indicative of concomitant systemic disease. To highlight the importance of thorough examination to rule out systemic disease, we present two childhood cases of orofacial granulomatosis, one of which was associated to Crohn's disease.
\end{abstract}

\section{Introduction}

Orofacial granulomatosis (OFG) is a chronic granulomatous condition characterized by relapsing and remitting lip swelling and oral involvement affecting the buccal mucosa, gingivae and floor of the mouth. ${ }^{1,2}$ Histologically, the condition is characterized by non-caseating granulomas that block lymphatics causing lymphoedema. Eventually, fibrosis may develop, making the swelling permanent. ${ }^{3}$ Differential diagnoses include granulomatous cheilitis, where lesions are limited to the lips, and Melkersson-Rosenthal Syndrome, which consists of the triad of persistent lip or facial swelling, recurrent facial paralysis and fissured tongue (Table 1). ${ }^{4}$

The precise etiology of OFG is unknown, although allergies, infections, genetic predisposition and immunological mechanisms have been suggested as causative agents..$^{5-7}$ Furthermore, OFG is seen as an independent entity, but also in conjunction with systemic conditions such as tuberculosis, sarcoidosis and Crohn's disease. ${ }^{8-10}$ To highlight the association between childhood OFG and systemic disease, we present two cases of OFG in children.

\section{Case Report \#1}

Case 1 was a 12-year old boy referred to us because of persistent swelling of the lips and gingivae, along with an uncharacteristic facial rash (Figure 1). He had a history of atopic dermatitis, but was otherwise previously healthy and had no predisposition to skin- or gastrointestinal disease. He presented with pronounced perioral edema, severe fissuring of the lips, angular cheilitis, gingival edema, and cobblestone formation of the buccal mucosa. During flare-ups the boy experienced severe abdominal pain. Suspecting inflammatory bowel disease, the patient was thoroughly examined with blood tests, biopsies from the gingival and buccal mucosa, and endoscopy of the gastrointestinal tract. The histopathological examination of the mucosal biopsies revealed chronic granulomatous inflammation consistent with OFG and oral Crohn's disease. Serum calprotectin was elevated to $409 \mathrm{mg} / \mathrm{kg}$. CRP and blood sedimentation rate were normal. Gastroscopy showed duodenal inflammation and by subsequent endoscopy ileac aphtous ulcers were detected. The patient was diagnosed with Crohn's disease. Infliximab treatment was initiated with a good clinical response on both abdominal symptoms and mucosal lesions.

\section{Case Report \#2}

Case 2 was a 9-year old boy who presented chronic swelling of the upper lip, redness, swelling and hypertrophy of the gingivae, and cobblestone formation of the buccal mucosa (Figure 2). This patient had IgE-mediated allergy to grass, birch, and alternaria, but was otherwise healthy and was not predisposed to any skin or systemic diseases. He had no history of abdominal pain or stool alteration and no respiratory symptoms. As in case 1, the mucosal biopsy from the upper-lip revealed granulomatous inflammation. All blood tests were normal. The patient was further conferred with a pediatric gastroenterologist. Serum and fecal calprotectin were normal. As this patient had no history of abdominal problems, no further examination was indicated at the time. The oral lesions were treated with topical fluticasone propionate 50 $\mathrm{g} /$ dose. The initial dosage was 1 spray unit twice daily. After 6 weeks this was reduced to once daily for another 6 weeks, after which the treatment was given pro necessitate. The lesions responded well to this treatment and after 12 weeks only mild infiltration and swelling of the gingivae remained without noticeable inflammatory activity. Regular check-ups every 3 months ensure that the condition is stable, and that possible gastrointestinal symptoms are detected.
Correspondence: Anne Birgitte Simonsen, Department of Dermatology, Aarhus University Hospital, Norrebrogade 44, 8000 Aarhus C, Denmark.

Tel. +45.784.50000

E-mail: anbsim@rm.dk

Key words: orofacial granulomatosis, children, Chron's disease.

Contributions: the authors contributed equally.

Conflict of interests: the authors declare no potential conflict of interests.

Received for publication: 20 August 2013.

Revision received: 17 November 2013.

Accepted for publication: 20 December 2013.

This work is licensed under a Creative Commons Attribution NonCommercial 3.0 License (CC BYNC 3.0).

(C) Copyright A.B. Simonsen and M. Deleuran, 2014 Licensee PAGEPress, Italy

Dermatology Reports 2014; 6:5039

doi:10.4081/dr.2014.5039

\section{Discussion}

Whether OFG should be considered a separate entity or a manifestation of systemic disease remains a topic of discussion. ${ }^{4}$ In particular, several case reports have suggested an association to Crohn's disease. The two entities share a number of clinical and histological features, but the exact relationship has not yet been established. ${ }^{4}$ Some authors suggest that patients with OFG may have subclinical Crohn's disease. ${ }^{1,11}$ Furthermore, several authors have observed that children are more likely to have onset of OFG preceding symptoms of Crohn's disease, ${ }^{3,11-13}$ and speculate that childhood onset carries a higher risk of developing Crohn's disease.

The prevalence of Crohn's disease in children with OFG is not well-established and could be underestimated since our current knowledge is based on case reports and case studies. ${ }^{3}$ The clinical presentation of the two patients presented here was almost identical. The history revealed that one of the patients had intermittent abdominal pain, which led to further examination, revealing an underlying Crohn's disease. This illustrates an important point, to be remembered when encountering children with OFG. The clinical presentation is not indicative of concomitant systemic disease and symptoms of this may be few. Regardless of the clinical presentation, systemic disease should always be ruled out. OFG has been reported to be a manifestation of tuberculosis and sarcoidosis, ${ }^{8-10}$ however, the vast majority of the literature focuses on the possible link 
Table 1. Differential diagnoses of orofacial granulomatosis.

\begin{tabular}{|c|c|c|c|c|c|c|}
\hline & $\begin{array}{l}\text { Granulomatous } \\
\text { swelling of lips }\end{array}$ & $\begin{array}{c}\text { Cobblestone formation } \\
\text { of oral mucosa }\end{array}$ & $\begin{array}{l}\text { Mucosal } \\
\text { swelling }\end{array}$ & $\begin{array}{l}\text { Oral } \\
\text { ulcers }\end{array}$ & $\begin{array}{c}\text { Facial nerve } \\
\text { paralysis }\end{array}$ & $\begin{array}{l}\text { Plicated } \\
\text { tongue }\end{array}$ \\
\hline Orofacial granulomatosis & + & + & + & $(+)$ & - & - \\
\hline Granulomatous cheilitis & + & - & - & - & - & - \\
\hline Melkersson-Rosenthal Syndrome & + & - & - & - & + & + \\
\hline
\end{tabular}

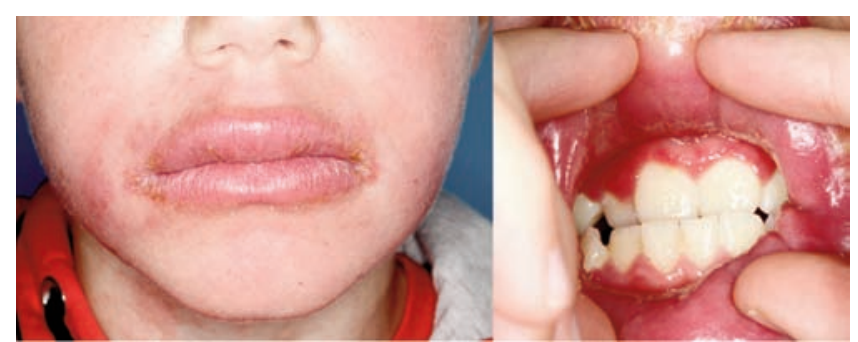

Figure 1. Perioral edema, severe fissuring of the lips, angular cheilitis, gingival edema, and cobblestone formation of the mucosa.

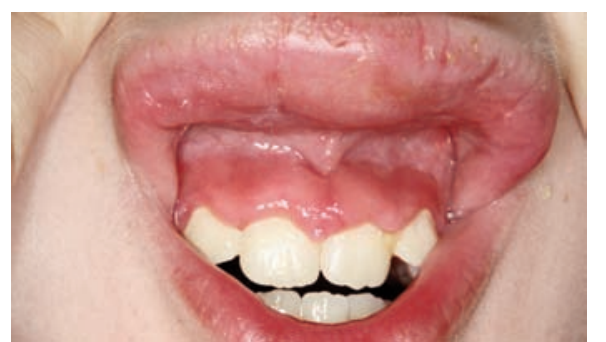

Figure 2. Chronic swelling of the upper lip. Redness, swelling and hypertrophy of the gingivae. between OFG and crohn's disease (Supplementary Table 1). ${ }^{3,11,12,14-20}$ In our opinion, the caring for pediatric patients with OFG should focus mainly on monitoring for signs, symptoms, and laboratory evidence of crohn's disease. A thorough patient history and clinical examination is necessary and we recommend blood screening including CRP, blood sedimentation rate, complete blood count, and serum calprotectin. If serum calprotectin is elevated, the patient should be referred to a pediatric gastroenterologist. Any specific symptoms or abnormal blood screening should lead to further examination of relevance, i.e. a history of pulmonary symptoms should lead to $\mathrm{X}$-ray examination of the chest and further blood tests depending on the symptoms and patient history. We recommend that patients be followed closely until the oral symptoms stabilize. When the condition is stable follow up at regular intervals ensures that the condition remains stable and any new symptom of systemic disease is detected.

\section{References}

1. Sanderson J, Nunes C, Escudier M, et al. Oro-facial granulomatosis: Crohn's disease or a new inflammatory bowel disease? Inflamm Bowel Dis 2005;11:840-6.

2. Campbell H, Escudier M, Patel P, et al. Distinguishing orofacial granulomatosis from crohn's disease: two separate disease entities? Inflamm Bowel Dis 2011;17:210915.

3. Tuxen AJ, Orchard D. Childhood and ado- lescent orofacial granulomatosis is strongly associated with Crohn's disease and responds to intralesional corticosteroids. Australas J Dermatol 2010;51:124-7.

4. Tilakaratne WM, Freysdottir J, Fortune F. Orofacial granulomatosis: review on aetiology and pathogenesis. J Oral Pathol Med 2008;37:191-5.

5. Kemmler N, Pfannschmidt N, Strohal R. Orofacial granulomatosis as first manifestation of Crohn's disease: successful treatment of both conditions with a combination of infliximab and dapsone. Acta Derm Venereol 2012;92:406-7.

6. Grave B, McCullough M, Wiesenfeld D. Orofacial granulomatosis - a 20-year review. Oral Dis 2009;15:46-51.

7. Campbell HE, Escudier MP, Patel P, et al. Review article: cinnamon- and benzoatefree diet as a primary treatment for orofacial granulomatosis. Aliment Pharmacol Ther 2011;34:687-701.

8. Al-Azri AR, Logan RM, Goss AN. Oral lesion as the first clinical presentation in sarcoidosis: a case report. Oman Med J 2012;27:243-5.

9. Ramesh V. Orofacial granulomatosis due to tuberculosis. Pediatr Dermatol 2009;26:108-9.

10. Kruschinski C, Welkoborsky HJ. Tuberculosis of the larynx associated with orofacial granulomatosis in childhood. Otolaryngol Head Neck Surg 2005;132:9679.

11. Saalman R, Mattsson U, Jontell M. Orofacial granulomatosis in childhood-a clinical entity that may indicate Crohn's disease as well as food allergy. Acta Paediatr 2009;98:1162-7.
12. Khouri JM, Bohane TD, Day AS. Is orofacial granulomatosis in children a feature of Crohn's disease? Acta Paediatr 2005;94:501-4.

13. Sainsbury CP, Dodge JA, Walker DM, Aldred MJ. Orofacial granulomatosis in childhood. Br Dent J 1987;163:154-7.

14. Rana AP. Orofacial granulomatosis: a case report with review of literature. J Indian Soc Periodontol 2012;16:469-74.

15. Smith VM, Murphy R. Orofacial granulomatosis: three case reports illustrating the spectrum of disease and overlap with Crohn's disease. Clin Exp Dermatol 2013;38:33-5.

16. Girlich C, Bogenrieder T, Palitzsch KD, et al. Orofacial granulomatosis as initial manifestation of Crohn's disease: a report of two cases. Eur J Gastroenterol Hepatol 2002;14:873-6.

17. Kolho KL, Heiskanen K, Verkasalo M, Pitkaranta A. Orofacial granulomatosis in children: a challenge for diagnosis and treatment. Int J Pediatr Otorhinolaryngol 2011;75:864-7.

18. Singhal P, Chandan GD, Das UM, Singhal A. A rare case report of orofacial granulomatosis in a pediatric patient. J Indian Soc Pedod Prev Dent 2012;30:262-6.

19. Kaarthikeyan G, Arvind M, Jayakumar N, Khakar M. Idiopathic orofacial granulomatosis in a young patient: a rare entity. $\mathrm{J}$ Oral Maxillofac Pathol 2012;16:432-4.

20. Howell JL, Bussell RM, Hegarty AM, Zaitoun H. Service evaluation of patients with orofacial granulomatosis and patients with oral Crohn's disease attending a paediatric oral medicine clinic. Eur Arch Paediatr Dent 2012;13:191-6. 